\title{
Package Delivery Using Autonomous Drones in Skyways
}

\author{
Woojin Lee \\ The University of Sydney \\ Australia \\ Balsam Alkouz \\ The University of Sydney \\ Australia
}

\author{
Babar Shazaad \\ The University of Sydney \\ Australia
}

\author{
Athman Bouguettaya \\ The University of Sydney \\ Australia
}

wlee2926@uni.sydney.edu.au balsam.alkouz@sydney.edu.aubabar.shahzaad@sydney.edu.authman.bouguettaya@sydney.edu.au

\begin{abstract}
Current drone delivery systems mostly focus on point-to-point package delivery. We present a multi-stop drone service system to deliver packages anywhere anytime within a specified geographic area. We define a skyway network which takes into account flying regulations, including restricted areas and no-fly zones. The skyway nodes typically represent building rooftops which may act as both recharging stations and delivery destinations. A heuristic-based $A^{*}$ algorithm is used to compute an optimal path from source to destination taking into account a number of constraints, including delivery time, availability of recharging stations, etc. We deploy our drone delivery system in an indoor testbed environment using a 3D model of Sydney CBD. We describe a graphical user interface to monitor the real-time package delivery in the skyway network.
\end{abstract}

\section{CCS CONCEPTS}

- Computer systems organization $\rightarrow$ Robotic autonomy; • Applied computing $\rightarrow$ Service-oriented architectures.

\section{KEYWORDS}

Drone delivery; Skyway network; Recharging constraint

\section{ACM Reference Format:}

Woojin Lee, Balsam Alkouz, Babar Shazaad, and Athman Bouguettaya. 2021. Package Delivery Using Autonomous Drones in Skyways. In Conference on Pervasive and Ubiquitous Computing. ACM, New York, NY, USA, 2 pages. https://doi.org/10.1145/1122445.1122456

\section{INTRODUCTION}

Drones are unmanned aircraft that operate with various degrees of autonomy. They have seen phenomenal growth and uptake which has come as a result of the drop in their prices and increased sophistication [3]. The wide availability of drones opens opportunities for a wide number of applications, such as emergency response, surveillance, and package delivery [7]. Using drones in delivery services is a fast-growing industry that gained a lot of commercial attention from companies such as Amazon, DHL, and Google [2]. COVID-19 pandemic has highlighted the need for such technologies as drones to ensure that economic activities continue to thrive. Drones provide a safer, socially distanced, contactless,

Permission to make digital or hard copies of all or part of this work for personal or classroom use is granted without fee provided that copies are not made or distributed for profit or commercial advantage and that copies bear this notice and the full citation on the first page. Copyrights for components of this work owned by others than ACM must be honored. Abstracting with credit is permitted. To copy otherwise, or republish, to post on servers or to redistribute to lists, requires prior specific permission and/or a fee. Request permissions from permissions@acm.org.

Ubicomp21, Sep 21-26, 2021, Virtual

(c) 2021 Association for Computing Machinery.

ACM ISBN 978-x-xxxx-xxxx-x/YY/MM. \$15.00

https://doi.org/10.1145/1122445.1122456

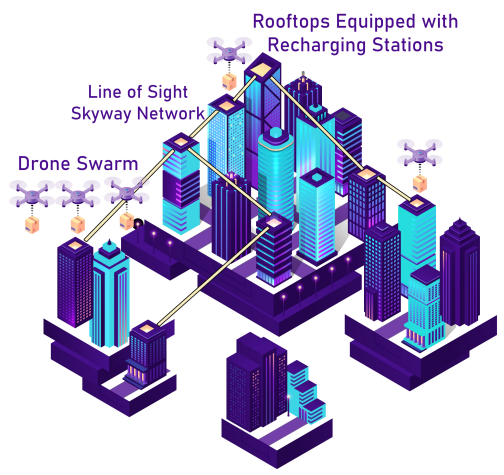

Figure 1: Drone Delivery in a Skyway Network

and more resilient alternative to deliver goods in cities and regional areas [5].

This demo focuses on developing a novel system to effectively provision drone-based delivery services in a skyway network. A skyway network enables the safe and scalable deployment of dronebased delivery solutions in shared airspace [6]. Fig. 1 depicts the drone delivery in a skyway network where nodes are the building rooftops. We use existing infrastructure of a city where each building rooftop may be easily and cheaply fitted with a recharging pad.

\section{DEMONSTRATION SYSTEM}

\subsection{Environment and Hardware Components}

Having an outdoor test environment is fraught with risks because of government restrictions when using drones in built-up areas [4]. Therefore, we setup a 3D model of Sydney CBD as an indoor drone testbed at the University of Sydney (Fig. 2). We build a skyway network which contains virtual barriers to prevent drones' access to no-fly zones and restricted areas. A skyway network is defined as a set of connected nodes representing take-off and landing stations. Each node may concurrently act as a recharging station. We use the LoS-based paths computed in subsection 2.2.1 to perform dronebased deliveries in the skyway network from a given source to a destination. We use a DfI Tello EDU drone that is safe and well suited to fly in an indoor testbed for its small size and robust nature.

\subsection{Software Components}

The software consists of four components as follows. The first component computes the LoS paths among all building rooftops to construct a skyway network. Then, the second component computes an optimal path from a given source to a destination using a heuristic-based $A^{*}$ algorithm. The third component is used to navigate the drone following the LoS paths in the skyway network. Finally, the fourth component presents a graphical user interface to monitor the drone position and battery status during the delivery operation. 




Figure 2: 3D Model of Sydney CBD

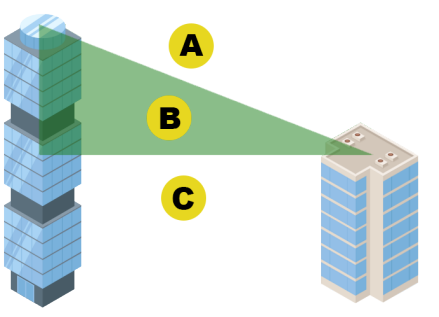

Figure 3: Line of Sight Intersection

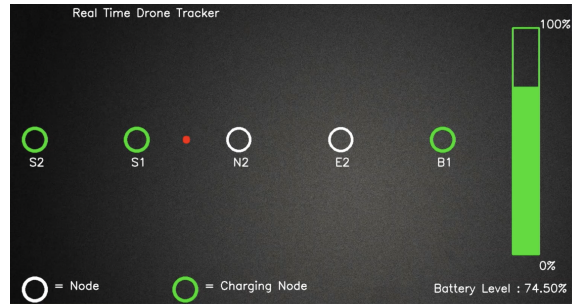

Figure 4: GUI Screenshot
2.2.1 Skyway Network. We construct a skyway network using the $(\mathrm{x}, \mathrm{y}, \mathrm{z})$ coordinates of the building rooftops. The skyway network takes into account the drone flying regulations, including flying within LoS and avoiding no-fly zones. The LoS is defined as a direct line segment between any two building rooftops having no barrier in between. We develop a LoS algorithm to compute the LoS paths between building rooftops considering no-fly zones. The no-fly zones are usually defined by aviation authorities for security and public safety, e.g., CASA in Australia ${ }^{1}$. We specify seven no-fly zones where drones are not permitted to fly (as shown in Fig. 1).

The LoS algorithm detects barriers (i.e., intersecting buildings) between any two nodes (i.e., building rooftops) to form a skyway path between them. We first construct a rectangular polygon to represent a path between any two nodes taking into account the drone width. Then, we discover and mark all nodes intersecting with the rectangular polygon considering only the xy-plane. The initial two steps are used to reduce the search space and computation cost by selecting only feasible nodes. We then construct a right angle triangle between any two buildings using their heights which are represented by z-axis (as shown in Fig. 3). The yellow circled nodes indicate nodes which intersect with the rectangular polygon considering their $\mathrm{z}$-axis. The nodes above than the right angle triangle obstruct the LoS path (e.g., node A in this scenario). While the nodes below or within the right angle triangle do not obstruct the LoS path (e.g., node B and node C). We define a LoS-based straight path between any two nodes if no node obstructs the path. This process continues until all possible LoS paths are computed and connected to form the skyway network.

2.2.2 LoS Path Composition. We compute a temporal optimal path from the source to the destination using a heuristic-based $A^{*}$ algorithm [1]. In this context, temporal optimal path refers to a path that leads to the destination faster. The drone may need recharging if it cannot reach the destination with its current battery capacity. In such a case, we compose a set of LoS paths leading the package to the destination including intermediate recharging stations.

2.2.3 Flight Navigation. We implement our drone delivery system and navigate an autonomous flight using the DJI Tello EDU drone. The Tello EDU comes with a set of mission pads to make precise movements. Each mission pad contains a unique ID pattern for its identification. At the source, initial position coordinates of the drone are set to $(0,0,0)$. Then, the drone detects the next node position in the path computed using the optimal path composition (subsection 2.2.2). If the drone is not facing towards the next node,

${ }^{1}$ https://www.casa.gov.au/drones/drone-rules/flying-near-emergencies-and-publicspaces

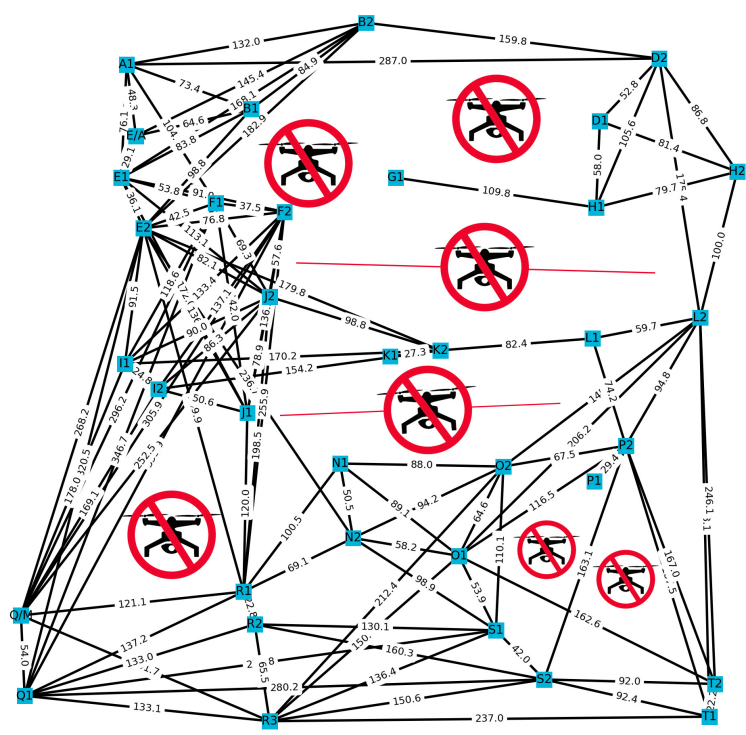

Figure 5: A Skyway Network with No-fly Zones it rotates in the direction of next node and travels over the LoS path. This navigation process continues until all the nodes in the optimal path are visited and package is delivered to the destination.

2.2.4 Graphical User Interface (GUI). We create a simple GUI to monitor the drone position during the delivery operation. The GUI prompts the user to input the source and the destination. Then, the GUI presents an optimal path to the destination along with drone's current position and battery status (Fig. 4).

\section{ACKNOWLEDGMENT}

This research was partly made possible by DP160103595 and LE180100158 grants from the Australian Research Council. The statements made herein are solely the responsibility of the authors.

\section{REFERENCES}

[1] Balsam Alkouz, Athman Bouguettaya, and Sajib Mistry. 2020. Swarm-based droneas-a-service (sdaas) for delivery. In IEEE ICWS. 441-448.

[2] Dane Bamburry. 2015. Drones: Designed for product delivery. Design Management Review 26, 1 (2015), 40-48.

[3] Grzegorz Chmaj and Henry Selvaraj. 2015. Distributed processing applications for UAV/drones: a survey. In Progress in Systems Engineering. Springer, 449-454.

[4] Therese Jones. 2017. International commercial drone regulation and drone delivery services. Technical Report. RAND.

[5] Adarsh Kumar et al. 2021. A drone-based networked system and methods for combating coronavirus disease (COVID-19) pandemic. FGCS 115 (2021), 1-19.

[6] Babar Shahzaad, Athman Bouguettaya, Sajib Mistry, and Azadeh Ghari Neiat. 2019. Composing drone-as-a-service (daas) for delivery. In IEEE ICWS. 28-32.

[7] Hazim Shakhatreh et al. 2019. Unmanned aerial vehicles (UAVs): A survey on civil applications and key research challenges. IEEE Access 7 (2019), 48572-48634. 